\title{
Epidural catheter migration and extra-spinal drug delivery: a possible cause of inflammation and/or infection
}

\author{
Maria Angeles Rodríguez Navarro', Francisco Miguel González Valverde ${ }^{3 *}$, Maria Victoria Redondo Carazo ${ }^{2}$, Jose A. Pérez Moreno ${ }^{1}$, \\ Martín García Muñoz', Jesus Alonso Castillo' and Mayo Saturno Marcos \\ *Correspondence: migova@terra.es \\ 'Department of anesthesiology, university hospital reina sofía, murcia, Spain. \\ ${ }^{2}$ Department of radiodiology, university hospital reina sofía, murcia, Spain. \\ ${ }^{3}$ Department of surgery, university hospital reina sofía, murcia, Spain.
}

\begin{abstract}
Purpose: There is a strong possibility that many problems associated with local anaesthetics go undocumented. We describe an epidural catheter complication and its consequence.

Clinical features: Female 56 year old patient operated 45 days previously for open gastric by-pass surgery and re-admitted to hospital because of pain and an infection related with the epidural technique. Lumbar Magnetic Resonance Imaging (MRI) revealed paravertebral inflammatory/infectious changes from L1 to L5, after lumbar epidural and post-operative epidural drug delivery for analgesia control. Intravenous antibiotic treatment and bed rest was continued for 21 days and a control MRI pointed to an improvement in the para-spinal alterations. There were never any signs of neurological deficit. We analyze, consider and discuss the clinical presentation of infectious complications of epidural anaesthesia, evaluating the various factors which might have led to this complication, as a silent migration of the catheter to a para-spinal region and the unintentional delivery of the drug involved, a foreign body reaction to the catheter tip or a result of the inflammatory process.

Conclusions: An early diagnosis based on a combination of physical examination, imaging studies and appropriate follow up of those patients would avoid severe complications. In order to reduce the risk of serious infection following epidural anesthesia, the clinician must be knowledgeable in the pathogenesis of these infections, and use of meticulous aseptic technique and post operative regular assessment of the patient (as a routine) to look for effective analgesia, motor block, catheter migration etc. The right fixed-catheter manoeuvre and use of the correct pumps delivery system are clearly indicated. Treatment must be initiated as soon as there is any suspicion of an infection related with a spinal technique and that prevention is the best alternative.
\end{abstract}

Keywords: Anaesthetic techniques, epidural anaesthesia, complications, epidural catheter migration, spinal infection, aseptic technique, anaesthesia for morbid obesity, equipment, catheter fixation

\section{Introduction}

Epidural complications such as paraplegia, nerve injury, haematoma, infections and catheter migration, have traditionally been considered rare, but recent studies suggest that their incidence has now reached levels of $0.1-0.125 \%$. [1-3]. The key to diagnosis of an infectious complication is having a high index of suspicion and the presence of patient risk factors (advanced age, nasal carriers, smoking, diabetes, immunocompromised status, and obesity). The most common presenting symptoms include backache, fever and headache but also neurologic deficits as rigors, neck pain and stiffness, radicular pain, lower extremity weakness, sensory deficits, bowel or bladder dysfunction, and paralysis. However, these signs and symptoms are not always present, have poor specificity, and need to be used in conjunction with laboratory and imaging tests (spinal MRI) to make the appropriate diagnosis. Laboratory tests may include a combination of blood, wound, and cerebrospinal fluid cultures, white blood cell count, erythrocyte sedimentation rate (ESR), and C-reactive protein (CRP) analysis. Differential diagnoses include meningitis, epidural hemorrhage, Anterior
Spinal Artery Syndrome, vertebral osteomyelitis, intervertebral disc space infections, other spinal canal infections and adjacent soft-tissue infections.

Present day protocols recommend immediate treatment when there is a suspicion of infection related with spinal techniques, while complications associated with epidural catheters should be prevented or, at least minimised, by handling aseptically and using methods to fix them in place that prevent their migration $[3,4]$. Lately, we use multimodal analgesia -combined epidural and intravenous drugs deliveryin order to avoid side-effects of opiods use but we also can hide incorrect function of one of them. The lack of surveillance data (incident pain, extent of block, etc...) further prevents any complications from inadequate function of the postoperative analgesia technique, and they could be the first signal of it.

\section{Case report}

We present a female 56 year old patient with antecedents of chronically high arterial pressure, morbid obesity, fibromyalgia, anxiety-depression syndrome, chronic back pain, non-alcoholic 


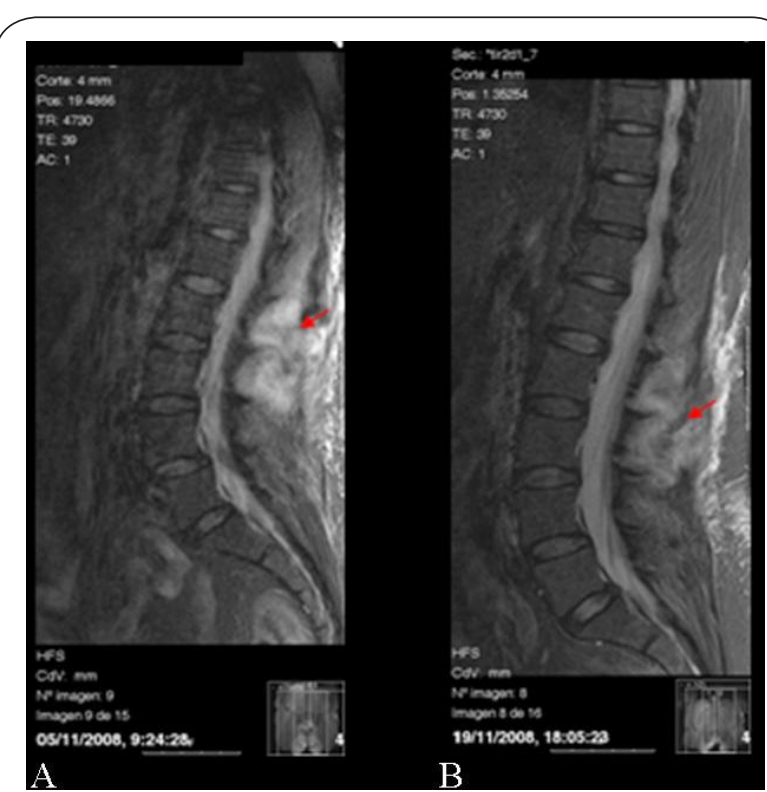

Figure 1. "Lumbar Magnetic Resonance Imaging: posterior infectious/inflammatory process from L1 to L5. Free liquid in subcutaneous cell space that might correspond to extravasated anaesthetic. No evidence of abscesses".

steatohepatitis and grade I aesophagitis. She was taking a chronic treatment with ibuprofen and anxiolytics. The patient had been operated 45 days previously for open gastric bypass and was re-admitted to hospital because of an infection related with the epidural technique.

The surgical intervention had been carried out under general anaesthetic and lumbar epidural. For this, a Portex ${ }^{\oplus}$ Thuoy 18 gauge needle was inserted at L2-L3 with the patient in the sitting position. The catheter (18 gauge closed-end multiport: Portex ${ }^{\circledR}$ Nylon Catheter) was threaded without difficulty; the distance from the skin to the epidural space was $8 \mathrm{~cm}$, and the length of the catheter remaining within the epidural space was $6 \mathrm{~cm}$. The catheter was fixed in place by a Tegaderm ${ }^{\circledast}$ dressing. An analgesic perfusion containing $0.8 \%$ levobupivacaine was administered intraepidurally for 76 hours following the operation by means of an elastomeric pump ( $10 \mathrm{ml} /$ hour), accompanied by continuous intravenous perfusion with dexketoprofen (150mg/24h). Probably, from the second postoperative day on the epidural block was not effective. Analogical visual pain evaluation scale (EVA) was daily obtained and the woman interviewed ranked her pain as a 4 on a scale of 0 to 10 which was localized in the back and was treated with $1000 \mathrm{mg}$ of acetaminophen. Our current multimodal postoperative-analgesia standard combines epidural analgesia and intravenous use of no steroidal antiinflammatory drugs. On the third postoperative day the epidural catheter was withdrawn by a nurse trained in epidural catheter removal. The nurse documented the procedure and assessed the catheter integrity. The catheter tip was sent for bacterial examination following the protocols of our anaesthesia department. Catheter culture resulted negative. The patient showed no undue symptoms (redness, swelling and tenderness near the insertion point or loss of functions) and the dressing was intact. She was discharged six days after the operation.

After attending the Emergency department twice because of back pain, the patient was seen by the Chronic Pain Unit of the hospital forty-five days after the operation to evaluate the continuous pain she felt in the region of the epidural insertion. Examination pointed to suppuration in the epidural puncture zone without a palpable swelling, while an emergency MRI of the dorso-lumbar column (Figure 1) revealed an abnormal signal for the posterior soft parts from L1 to L5. The image was described as a posterior infectious/ inflammatory process from L1 to L5, more acute in L2 and L3. Free liquid was noted in the subcutaneous tissue which might correspond to extravasated anaesthetic but there was no evidence of abscesses.

The patient was readmitted with a diagnosis of infection associated with the epidural technique. We consulted with an infectious disease specialist to facilitate initiation of early and effective therapy. Intravenous antibiotic treatment was initiated $(2 \mathrm{~g} / 12 \mathrm{~h}$ ceftazidime and $500 \mathrm{mg} / 6 \mathrm{~h}$ vancomycine) and analgesia with desketoprofen $50 \mathrm{mg} / 8 \mathrm{~h}$. The patient was never feverish and the insertion point ceased to suppurate the day of admission. Cultures of the transudate and blood were negative. C-reactive protein (C-RP) upon admission was $9 \mathrm{mg} / \mathrm{dl} \%$ (normal values $<0.3 \mathrm{mg} / \mathrm{dL}$ ). C-RP is synthesized in the liver in response to inflammation, tissue injury, infection, and malignancy, and CRP levels may be a more sensitive indicator of infection than other biochemical markers used.

Antibiotic treatment and bed rest was continued for 21 days and a control MRI was carried out which pointed to the continued presence of a similar amount of free liquid in the subcutaneous tissue, no abscesses (no requiring surgical drainage) and an improvement in the other para-spinal alterations. The patient was observed carefully for signs of infection but she recovered well and there were never any signs of neurological deficit.

Sixteen weeks after the epidural injection, including six weeks of antibiotic treatment, a new MRI pointed to the resolution of the infectious/inflammatory processes.

\section{Discussion}

The benefits of epidural analgesia have been demonstrated in many studies that defend the use of post-operation pain control (major abdominal surgery, thorax operations, knee prosthesis, etc), in major abdominal surgeries and for the relief of chronic pain. These studies have focused on the reduction of morbidity related with pain control, improving ventilation conditions, rapid rehabilitation and other benefits, such as diminishing the risk of bleeding and thrombosis $[1,4]$. The benefits of the epidural technique seem convincing, 
but some studies have failed to show improved effect when using perioperative epidural analgesia after gastric bypass surgery [5], while reminding readers that it is not a technique exempt from risks such as epidural-related infection, potential neurological complications, placement failure, and other technical complications $[3,4]$. These inconsistent results have created controversy regarding the role of epidurals in patient outcome. Patients suffering morbid obesity about to receive open gastric by-pass surgery are at particular risk since the morbidity-mortality of the anaesthetic/surgical process is high. This has led to a study of different anaesthetic techniques and epidural anaesthesia is at present controversial [6].

On the other hand, because intrathecal drug delivery transports pain medication directly to the receptors in the spinal cord, patients may achieve pain control with smaller dosages compared to oral pain medications, thereby likely reducing side effects. In our hospital, intrathecal drug delivery systems are implanted for chronic pain when conservative therapies have failed, surgery is ruled out, no active or untreated addiction exists, psychological testing indicates appropriateness for implantable therapy, medical contraindications have been eliminated (coagulopathies, infections), and a successful intrathecal drug trial has been completed. For major abdominal surgeries with extensive incisions, we opine that epidural infusions with local anaesthetic provide superior pain relief as compared with intrathecal or conventional parenteral narcotics. With less extensive surgery, however, intrathecal narcotics alone can be used for postoperative analgesia. A single dose of intrathecally (spinal) administered narcotic can provide substantial pain relief up to 18 to 24 hours postoperatively.

However, there are existing studies as well as reports in literature regarding the use of epidural analgesia for bariatric surgeries [7-10]. The literature points to several potential complications in this respect, including the inherent difficulty of technique or it can be time-consuming in obese patients [8], the possibility of infection $[2,9]$ or migration of the catheter itself [11-13].

Simpson and Al-Makadama's study on the epidural infusion of drugs and spinal infection [3] evaluates all the above data and emphasises the fact that spinal infections may be very difficult to diagnose since symptoms of neurological deficits appear late, or, as in our case, does not appear. Diagnosis typically takes an average of one month, but can take as long as six months, impeding effective and timely treatment. Many patients do not seek medical attention until their symptoms become severe or debilitating. For this reason they emphasise the importance of prevention and insist that, in the case of any clinical doubt, an MRI should be carried out and antibiotics prescribed. Only $13 \%$ of patients suffering spinal infection manifest the triad of high temperature, back pain and neurological alterations, while $75 \%$ only feel back pain at the outset and $66 \%$ pyrexia. Two out of three patients have leucocytosis and C-RP levels may be normal.
In the case described here, the epidural catheter had been placed with maximum asepsis after correctly preparing the skin with antiseptic. In obese population, epidural placement failure is relatively frequent because of difficulty in identifying anatomic landmarks and the need for longer needles (and hence to sacrifice tactile cues). Even after successful placement, inadequate epidural function requiring catheter replacement is more common in obese than non-obese patients, possibly because of increased mobility of overlying excess soft tissue. Therefore, we left the catheter deeper than usual. The catheter was connected to an infusion pump in the operating theatre and in the resuscitation unit to an elastomeric pump prepared by the hospital pharmacy service (again in conditions of maximum sterility). This was maintained for 72 hours at 10 $\mathrm{ml} / \mathrm{h}$ and was at no moment further manipulated. We prefer the use of elastomeric pumps instead of infusion electronic pumps because this system is secure (can not be manipulated by the patient or people without experience) and avoids any other drugs infusion by epidural catheter. Nevertheless, elastomeric pumps do not have any system to prevent and alert of others "drugs delivery errors" as catheter displacement, increased/decreased pressures or even occlusion as we could obtain with infusion pumps or patient controlled analgesia system (PCA).

The patient began to complain of back pain while she was still in hospital (EVA score of 4) but probably because of her chronic back pain and fibromyalgia, the pain was misinterpreted as an osteo-muscular pain. We explore the punction area everyday looking for undue symptoms as swelling, redness, tenderness or suppuration. Examination of the back of a morbidly obese patient is difficult but no noticeable changes were registered. We also perform an exam to look for effective analgesia, motor block, catheter migration or a loss of functions such as movement or sensation.

After the MRI the patient was admitted with a suspected spinal infection. Antibiotics were prescribed, according to the above mentioned paper [3]. The aetiology of paraspinal infection may be due to bacterial colonization of the catheter or puncture, although in a morbidly obese patient other differential diagnosis should be borne in mind: 1. Catheter migration -perhaps later during the postoperative periodcausing partial delivery of the drug to subcutaneous space, 2. Possible foreign body reaction to a retained tip or catheter material, 3. Possible infection related to the introduction of catheter with a later manifestation.4. A fluid collection caused by a reaction to the inflammatory process.

Spinal infections often require long-term intravenous antibiotic or antifungal therapy and can equate to extended hospitalization time for the patient. Immobilization may be recommended when there is significant pain or the potential for spine instability. If the patient is neurologically and structurally stable, antibiotic treatment should be administered after the organism causing the infection is properly identified. Patients generally undergo antimicrobial therapy for a 
minimum of six to eight weeks. The type of medication is determined on a case-by-case basis depending on the patient's specific circumstances, including his or her age. Surgery may be indicated when significant bone involvement, neurological deficits, sepsis with clinical toxicity or failure of intravenous antibiotics alone to eradicate the infection is present. Current treatment protocols for spinal infections require treatment by a multidisciplinary team of physicians, including infectious disease experts, neuroradiologists, and spine surgeons. The team will be able to assess the best treatment approach on an individualized basis, whether it is surgical or nonsurgic.

The continuous infusion of the drug dispensed by the elastomeric pump at $10 \mathrm{ml} / \mathrm{h}$ for 72 hours, with the probably extra-epidural diffusion of a portion of the liquid and the inflammatory/infectious response to the same may have been the origin of the complication. Extravasations of local anaesthetic may result infected, but more often the possibility of damage by compression or inflammation of the adjacent tissue and myotoxicity with breakdown of muscle fibers. In addition to muscle fiber damage, the common pathologic feature seen in this condition is an early damage, whether direct or indirect, to the vasculature in the area of injection. This frequently results in pockets of haemorrhage and also in a focus of ischemic necrosis.

Although we cannot affirm that this was the cause, nor in what stage of the post-operation period the migration might have occurred, this possibility should be considered when analysing the MRI. Against the possibility that the origin was unnoticed extra-epidural infusion was the fact that, 45 days had already elapsed since the operation, time enough for the liquid to have been re-absorbed. However, in favour of this diagnosis is the serious alteration of the soft tissue suggested by the MRI and the presence of a large quantity of free para-spinal liquid, together with the poor symptomology and absence of fever, which does not correspond to infectious signs but to diffusion of the infused liquid. The patient already complained of back pain before being discharged, while the pain of the wound might have been mitigated by the desketoprofen. The multimodal perioperative analgesia combining epidural analgesia and intravenous nonsteroidal anti- inflammatory drugs (habitually desketoprofen) avoiding full use of opiates but in this patient this strategy could dress up that epidural did not work properly.

Another look at the bibliography reveals that epidural catheter migration is a frequent complication: in postoperative analgesia, for example, up to $50 \%$ of catheters move [11-13], which has led to the development of different fixation systems, and to debate as to how best carry out post-operative controls of where the tip is placed either by radiology or other procedures. Migration to the intradural space, fragmentation, displacement in a caudal or cranial direction may involve fatality [12-15].

As regards the rate of epidural infusion (in the present case, $10 \mathrm{ml} / \mathrm{h}$ ), comparative studies between two options - small volumes at high concentrations or large volumes at low concentrations - there is not much difference since the quality of analgesia depends on the total dose administered [16]. However, when post-operative perfusion continues for more than 72 hours we should pause to think whether such a rate is suitable given the high frequency of catheter migration described in. If $900-1000 \mathrm{ml}$ of anaesthetic liquid is infused inadvertently outside the epidural space, it can become over-infected or, more probably, it causes myotoxicity and neurological symptoms through compression as it accumulates near the spine. Nonetheless, physician vigilance in monitoring and recognizing those complications is crucial in determining outcomes and cannot be overemphasized.

Which make this case unique is the simultaneity of several aspects that we must consider in the future of neuraxial analgesia in obese patients: pain symptoms of fibromyalgia should be differentiated from specific one; meticulous aseptic technique for the placement and manipulation of the catheter is mandatory; the use of system to fix epidural catheter is essential to avoid migration; elastomeric pumps don't warn us about an incorrect function and multimodal analgesia could hide pain. The key to resolve all of these is to test if epidural is providing adequate regional analgesia but also we need reject the possibility of infection, migration or another important complication.

The conclusions we can draw from an analysis of the present case is that the diagnosis of the epidural complications is based on a combination of clinical suspicion, physical examination, laboratory and imaging studies and appropriate follow up of this patients, especially when risk factors are present. Treatment must be initiated as soon as there is any suspicion of an infection related with a spinal technique (a move which might save lives) and that prevention is the best alternative - aseptic manipulation and the use of methods that hinder migration to the greatest extent possible. In this respect, we agree with Tornero JC et al., [17], in that there are relatively few publications dealing with complications associated with local anaesthetics, which suggest that they are infrequent, although there is strong possibility that many go undocumented.

\section{Competing interests}

The authors declare that they have no competing interests.

\section{Publication history}

Received: 20-Dec-2012 Revised: 06-Jan-2013

Re-Revised: 21-Jan-2013 Accepted: 02-Feb-2013

Published: 09-Feb-2013

\section{References}

1. Anim-Somuah $M$, Smyth $R$ and Howell C: Epidural versus non-epidural or no analgesia in labour. Cochrane Database Syst Rev 2005, CD000331. | Article | PubMed

2. Gosavi C: Epidural abscess complication insertion of epidural catheters. Br J Anaesth 2004, 92:294-5. I Article

3. Simson K and Said Al-Makadma Y: Epidural drug delivery and spinal infection. Continuing Education in Anaesthesia, Critical Care \& Pain 2007, 
Rodríguez Navarro et al. Journal of Anesthesiology and Clinical Science 2013, http://www.hoajonline.com/journals/pdf/2049-9752-2-11.pdf

7:112-5. | Article

4. Jorgensen $\mathrm{H}$, Wetterslev J, Moiniche $\mathrm{S}$ and Dahl J B: Epidural local anaesthetics versus opioid-based analgesic regimens on postoperative gastrointestinal paralysis, PONV and pain after abdominal surgery. Cochrane Database Syst Rev 2000, CD001893. | Article | PubMed

5. Ganapathy S, McCartney C J, Beattie W S and Chan V W: Best evidence in anesthetic practice: prevention: epidural anesthesia and analgesia does not reduce 30-day all-cause mortality and major morbidity after abdominal surgery. Can J Anaesth 2003, 50:143-6. | Article | PubMed

6. Otsu I, Thirlby RC and Allen HW: Patient controlled thoracic epidural analgesia after gastric bypass surgery for morbid obesity. Reg Anesth Pain Med 2006, 26: A117. | Article

7. Akbay B K, Hayit F, Pehlivan M and Agartan C: Anaesthetic management of an extremely obese woman undergoing open gastric bypass. Eur J Anaesthesiol 2006, 23:983-6. | Article | PubMed

8. Schumann R, Shikora S, Weiss J M, Wurm H, Strassels S and Carr D B: A comparison of multimodal perioperative analgesia to epidural pain management after gastric bypass surgery. Anesth Analg 2003, 96:469-74. | Article | PubMed

9. Yuan H B, Zuo Z, Yu K W, Lin W M, Lee H C and Chan K H: Bacterial colonization of epidural catheters used for short-term postoperative analgesia: microbiological examination and risk factor analysis. Anesthesiology 2008, 108:130-7. | Article | PubMed

10. Sasakawa T, Nagashima M, Hamada I, Takahata O, Sengoku K, Fujita S and Iwasaki H: [Delayed subarachnoid migration of an epidural catheter: a case report]. Masui 2004, 53:284-6. | Article | PubMed

11. Phillips $D C$ and Macdonald R: Epidural catheter migration during labour. Anaesthesia 1987, 42:661-3. . | Article | PubMed

12. Lang $S A$ and Arraf J: Analgesia in bariatric patients following upper abdominal surgery. Can J Anaesth 2004, 51:276; author reply 276. | Article I PubMed

13. Day $Y$ and Graham D: Epidural catheter migration. Anaesthesia 2002, 57:418. | Article | PubMed

14. Demiraran $Y$, Yucel I and Erdogmus B: Subcutaneous effusion resulting from an epidural catheter fragment. Br J Anaesth 2006, 96:508-9. | Article | PubMed

15. Visser W A: Delayed subarachnoid migration of an epidural catheter. $A n-$ esthesiology 1998, 88:1414-5. | Article | PubMed

16. Dernedde M, Stadler M, Bardiau F and Boogaerts J G: Continuous epidural infusion of large concentration/small volume versus small concentration/large volume of levobupivacaine for postoperative analgesia. Anesth Analg 2003, 96:796-801. | Article | PubMed

17. Tornero JC, Gómez $M$, Fabregat $C$, Aliaga $F$, Roqués $V$, Escamilla $A$ and Guerrí Cebollada A: Complicaciones tras técnicas de anestesia regional. Rev Esp Anestesiol Reanim 2008, 55: 552-563. I PDF

\section{Citation:}

Rodríguez Navarro M A, González Valverde F M, Redondo Carazo M V, Pérez Moreno J A, García Muñoz M, Castillo J A and Marcos M S: Epidural catheter migration and extra-spinal drug delivery: a possible cause of inflammation and/or infection. journal of Anesthesiology and Clinical Science 2013, 2:11. http://dx.doi.org/10.7243/2049-9752-2-11 